\title{
Confined variational calculation of positronium-hydrogen scattering below the positronium excitation threshold
}

\author{
M.-S. Wu $\odot,{ }^{1}$ J.-Y. Zhang $\odot,{ }^{1, *}$ Y. Qian $\odot,{ }^{2}$ K. Varga, ${ }^{3}$ U. Schwingenschlögl, ${ }^{4}$ and Z.-C. Yan ${ }^{5,1}$ \\ ${ }^{1}$ State Key Laboratory of Magnetic Resonance and Atomic and Molecular Physics, \\ Innovation Academy for Precision Measurement Science and Technology, Chinese Academy of Sciences, Wuhan 430071, China \\ ${ }^{2}$ School of Computer Science and Technology, East China Normal University, Shanghai 200062, China \\ ${ }^{3}$ Department of Physics and Astronomy, Vanderbilt University, Nashville, Tennessee 37235, USA \\ ${ }^{4}$ Physical Science and Engineering Division, King Abdullah University of Science and Technology (KAUST), \\ Thuwal 23955-6900, Saudi Arabia \\ ${ }^{5}$ Department of Physics, University of New Brunswick, Fredericton, New Brunswick, Canada E3B 5 A3
}

(Received 3 December 2020; accepted 2 February 2021; published 15 February 2021)

\begin{abstract}
The confined variational method is used to study $S$-, $P$-, and $D$-wave positronium-hydrogen scattering with the scattering energy between 0.068 and $3.333 \mathrm{eV}$. Accurate phase shifts and $S$-wave scattering lengths are calculated and compared with other theoretical methods. Existing discrepancies for the $D$-wave phase shifts are resolved. According to collision momentum, total orbital angular momentum, and spin configuration, the distortion effects of positronium are quantitatively studied. Finally, the finite nuclear mass effects are investigated.
\end{abstract}

DOI: 10.1103/PhysRevA.103.022817

\section{INTRODUCTION}

Positronium (Ps) is a two-body system composed of an electron and a positron. Since the composition of Ps is purely leptonic, it plays a significant role in testing the theory of quantum electrodynamics (QED) [1-5]. For example, the recent measurement of a fine-structure splitting in Ps has shown a puzzling discrepancy with the QED prediction [5]. In addition, the $\gamma$-ray lasers based on a Ps Bose-Einstein condensate have attracted considerable attention and have been extensively investigated [6-9]. The study of Ps-matter scattering is essential to understand the Ps-matter interaction [10-14], as well as the similarities and differences between matter-matter and matter-antimatter interactions [15-21]. Ps beam experiments with $\mathrm{He}, \mathrm{Ar}, \mathrm{Kr}, \mathrm{Xe}, \mathrm{H}_{2}, \mathrm{~N}_{2}, \mathrm{O}_{2}$, and $\mathrm{SF}_{6}$ have revealed electronlike scattering of Ps at intermediate energies [21].

There are many theoretical studies of Ps-H scattering using various approaches, including the Kohn variational method [22-24], the inverse Kohn variational method [22,23], the complex Kohn variational method [25,26], the diffusion Monte Carlo method [27], the stochastic variational method (SVM) [28,29], the close-coupling method [30-36], the static exchange method [37-39], the many-body theory [40], and the confined variational method (CVM) [41]. To handle the complicated short-range correlations in Ps-H scattering, correlated basis functions (Hylleraas-type basis [25], explicitly correlated Gaussian (ECG) basis [41], and others [24,27]) have been adopted in previous variational calculations.

To date, the most accurate elastic phase shifts for Ps-H scattering have been given by Walters et al. [30], Blackwood et al. [35], Zhang et al. [41], Woods et al. [25], and Wu et al. [45]. In

\footnotetext{
*jzhang@apm.ac.cn
}

the CVM calculation of Zhang et al., however, only the singlet and triplet $S$-wave phase shifts at the momenta $k=0.1$ and 0.2 a.u. are evaluated. The CVM phase shifts beyond $S$-wave scattering at higher energies need further investigation. In the CVM calculation of Wu et al., however, only the triplet $P$ wave phase shift at $k=0.1$ a.u. and the singlet $D$-wave phase shift at $k=0.4$ a.u. are evaluated. Accurate $S$ - and $P$-wave phase shifts with momentum up to $k=0.7$ a.u. are obtained by Woods et al. [25] using the complex Kohn variational method with Hylleraas-type correlation terms. However, the convergences of their singlet $D$-wave phase shifts at $k=0.2$ 0.7 a.u. are not as good as those of their $S$ - and $P$-wave phase shifts. Moreover, their extrapolations of the ${ }^{1} D$-wave phase shift at $k=0.1$ a.u. and all ${ }^{3} D$-wave phase shifts are not reliable [25].

The CVM approach, first proposed by Mitroy et al. [42] and further developed by Zhang et al. [41], is an ab initio method to study low-energy elastic scattering problems. It has been successfully applied to investigate $e-\mathrm{H}, e^{+}-\mathrm{H}, e-\mathrm{He}$, $e^{+}-\mathrm{He}, \mathrm{Ps}-\mathrm{H}$, and $\mathrm{Ps}_{-} \mathrm{H}_{2}$ scattering at $k \leqslant 0.2$ a.u. [41-44]. Recently, we have developed a strategy that can effectively eliminate nonphysical confinement effects while extending the CVM to non- $S$ partial wave scattering and scattering at higher energies [45]. In addition, contrary to the original $\mathrm{CVM}$, the strategy of using a smaller confining radius $R_{0}$ can greatly reduce the computational cost.

The purpose of the present work is to apply the CVM we have developed to extend our previous results on Ps- $\mathrm{H}$ scattering to higher partial waves at higher energies, and to provide more benchmark results for other theoretical methods. This paper is organized as follows. In Sec. II the advanced CVM is introduced. The computational results are presented in Sec. III, where the phase shifts for the Ps-H scattering of ${ }^{1,3} S_{-},{ }^{1,3} P_{-}$, and ${ }^{1,3} D$-waves below the Ps excitation threshold 
are given in Sec. III A, the distortion effects in Ps-H scattering are discussed in Sec. III B, and the calculations of the $S$-wave scattering lengths are given in Sec. III C. Finally, Sec. IV is a summary. Phase shifts are expressed in radians, and atomic units (a.u.) are used throughout unless otherwise stated.

\section{THEORY}

\section{A. Infinite nuclear mass}

For a many-body scattering problem, especially for a composite projectile and target, the scattering phase shift extracted directly from the scattering wave function usually has limited accuracy. The key idea of the CVM is that it converts an original many-body scattering problem into a modified manybody bound-state problem so that it can be solved by applying well-established bound-state techniques, and the scattering phase shift can then be extracted by solving an equivalent quasi-one-dimensional central-potential problem [42].

We first perform a many-body calculation by adding a confining potential $V_{\mathrm{cp}}$ to the Hamiltonian of the Ps-H system so that it becomes a bound-state eigenvalue problem,

$$
\begin{gathered}
\left(H+V_{\mathrm{cp}}\right) \Psi(\mathbf{r}, \mathbf{s})=E \Psi(\mathbf{r}, \mathbf{s}), \\
H=-\frac{1}{2} \sum_{i=1}^{3} \nabla_{i}^{2}+\sum_{i=1}^{3} \frac{Q q_{i}}{r_{i}}+\sum_{\substack{i, j=1 \\
i<j}}^{3} \frac{q_{i} q_{j}}{\left|\mathbf{r}_{j}-\mathbf{r}_{i}\right|},
\end{gathered}
$$

where the nuclear mass of hydrogen is assumed to be infinity, $\mathbf{r}_{1}, \mathbf{r}_{2}$, and $\mathbf{r}_{3}$ are, respectively, the position vectors of the two electrons and the positron relative to the fixed nucleus, $\mathbf{r}$ denotes $\left(\mathbf{r}_{1}, \mathbf{r}_{2}, \mathbf{r}_{3}\right)$ collectively, and $\mathbf{s}$ denotes $\left(\mathbf{s}_{1}, \mathbf{s}_{2}, \mathbf{s}_{3}\right)$ of the three lepton spins. Additionally, $q_{i}$ is the charge of the electron or positron, and $Q$ is the charge of the nucleus. $\Psi(\mathbf{r}, \mathbf{s})$ is the eigenfunction of $H+V_{\text {cp }}$ corresponding to $E$, where $E$ is the total energy of the original scattering system, including the bound-state energies of the projectile and target, as well as the kinetic energy of the projectile. The eigenfunction $\Psi(\mathbf{r}, \mathbf{s})$ can be expanded in terms of the ECG basis

$$
\phi_{n}(\mathbf{r}, \mathbf{s})=|\mathbf{v}|^{2 K+L} \exp \left(-\frac{1}{2} \mathbf{r}^{\mathrm{T}} A_{n} \mathbf{r}\right) Y_{L M}(\mathbf{v}) \chi(\mathbf{s}),
$$

where $\mathbf{v}=u^{\mathrm{T}} \mathbf{r}$, with $u^{\mathrm{T}}=\left(u_{1}, u_{2}, u_{3}\right)$ being a global vector, $\chi(\mathbf{s})$ is the spin function, $K$ is an integer that describes the number of nodes of the wave function, $A_{n}$ is a parameter matrix, $L$ and $M$ are, respectively, the total orbital angular momentum and its $z$ component, and $Y_{L M}$ is a spherical harmonics. The confining potential used here is

$$
V_{\mathrm{cp}}=\sum_{i=1}^{2} v_{\mathrm{cp}}\left(\rho_{i}\right)
$$

where

$$
v_{\mathrm{cp}}\left(\rho_{i}\right)= \begin{cases}0, & \rho_{i}<R_{0}, \\ G\left(\rho_{i}-R_{0}\right)^{2}, & \rho_{i} \geqslant R_{0} .\end{cases}
$$

$\rho_{i}=\left|\mathbf{r}_{i}+\mathbf{r}_{3}\right| / 2$ is the distance between the hydrogen nucleus and the center-of-mass of Ps, and $R_{0}$ is chosen to ensure that the complicated short-range interaction between Ps and $\mathrm{H}$ can be ignored outside the sphere of radius $R_{0}$. To eliminate unphysical confining effects, we define the following judgment index between two basis functions $\phi_{n}$ and $\phi_{m}$ :

$$
s_{i}^{n m}=\frac{\left\langle\phi_{n}\left|\Theta\left(r_{i 3}-R_{1}\right)\left(r_{i 3}-R_{1}\right)^{2}\right| \phi_{m}\right\rangle}{\left\langle\phi_{n}\left|\Theta\left(\rho_{i}-R_{0}\right)\left(\rho_{i}-R_{0}\right)^{2}\right| \phi_{m}\right\rangle},
$$

where $r_{i 3}=\left|\mathbf{r}_{i}-\mathbf{r}_{3}\right|$ is the distance between the electron and positron in a confined Ps, $R_{1}$ is an adjustable number greater than $2 a_{0}$, with $a_{0}$ being the Bohr radius, and $\Theta$ is the Heaviside step function. If the confining potential acts on the pseudo Ps formed by the positron and the electron of the hydrogen, $r_{i 3}$ will be much larger than the characteristic size $2 a_{0}$ of Ps. This means that $s_{i}^{n m}$ will be a large number when $R_{1}$ is set to $2 a_{0}$ or slightly larger. We discard $\left\langle\phi_{n}\left|V_{\mathrm{cp}}\left(\rho_{i}\right)\right| \phi_{m}\right\rangle$ when $s_{i}^{n m}$ exceeds a specific threshold. In this work, $R_{1}$ is set to be $15 a_{0}$, and the specific threshold for $s_{i}^{n m}$ is set to be 1.0.

The confining potential in Eq. (1) is tuned to ensure that a specific total energy is yielded. For example, if a groundstate projectile Ps of momentum $k=0.1$ is scattering with a ground-state target $\mathrm{H}$, then $E=-0.7475$. With the confining potential $v_{\mathrm{cp}}(\rho)$ thus determined, we then solve the one-dimensional bound-state problem

$$
\left(-\frac{1}{2 \mu} \nabla^{2}+V_{m}(\rho)+v_{\mathrm{cp}}(\rho)\right) \Phi(\rho)=E^{\prime} \Phi(\rho),
$$

where $\mu$ is the reduced mass between Ps and H, which is equal to 2 for the case of infinite nuclear mass, and $\Phi(\rho)$ is the eigenfunction with the associated eigenvalue $E^{\prime}$ that is equal to the scattering energy. Also in the above, $V_{m}(\rho)$ is the tunable model potential that has the form

$$
V_{m}(\rho)=\lambda e^{-\alpha \rho}-\frac{C_{6}}{\rho^{6}}\left(1-e^{-(\rho / \beta)^{6}}\right),
$$

where $\lambda, \alpha$, and $\beta$ are adjustable parameters, and $C_{6}$ is the van der Waals coefficient between $\mathrm{Ps}$ and $\mathrm{H}$ that is equal to 34.78473 [46]. It should be pointed out that the inclusion of $-C_{6} / \rho^{6}$ in $V_{m}(\rho)$ is to correctly describe the interaction between $\mathrm{Ps}$ and $\mathrm{H}$ in the asymptotic region [47], i.e., the region outside the sphere of radius $R_{0}$. In this work, we fix $\alpha=0.5$ and $\beta=5$, and we adjust $\lambda$ so that the bound-state problem Eq. (6) can yield the eigenvalue $E^{\prime}=k^{2} / 2 \mu$ for a given $k$. Finally, we solve the scattering equation for the potential $V_{m}(\rho)$ only,

$$
\left(-\frac{1}{2 \mu} \nabla^{2}+V_{m}(\rho)\right) \Phi^{\prime}(\rho)=E^{\prime} \Phi^{\prime}(\rho),
$$

by applying an integration procedure, and we determine the scattering phase shift $\delta_{\ell}(k)$ with a least-squares fit between $\Phi^{\prime}(\boldsymbol{\rho})$ and $A Y_{l m}(\hat{\boldsymbol{\rho}}) \sin \left[k \rho-\ell \pi / 2+\delta_{\ell}(k)\right] /(k \rho)$ for $\rho \rightarrow \infty$. It should be emphasized that the essence of these transformations from the original many-body scattering problem to Eqs. (1), (6), and then (8) is that the logarithmic derivatives of the wave functions determined by these equations are guaranteed to be equal to each other at the radial boundary $R_{0}$ [42].

\section{B. Finite nuclear mass}

For the case of finite nuclear mass, three main modifications should be made. The first modification is that the 
TABLE I. Comparison of $S$-wave phase shifts (in radians) among the present confined variational method (CVM), the complex Kohn method (cKohn) [25], the Kohn method (Kohn) [22], and the close-coupling method (CC, [30] for singlet and [35] for triplet), where $\delta_{0}^{+}$stands for spin singlet and $\delta_{0}^{-}$stands for spin triplet.

\begin{tabular}{lcccccccc}
\hline \hline$k$ & $\delta_{0}^{+}(\mathrm{CVM})$ & $\delta_{0}^{+}(\mathrm{cKohn})$ & $\delta_{0}^{+}(\mathrm{Kohn})$ & $\delta_{0}^{+}\left(\mathrm{CC}, 14 \mathrm{Ps}+14 \mathrm{H}+\mathrm{H}^{-}\right)$ & $\delta_{0}^{-}(\mathrm{CVM})$ & $\delta_{0}^{-}(\mathrm{cKohn})$ & $\delta_{0}^{-}(\mathrm{Kohn})$ & $\delta_{0}^{-}(\mathrm{CC}, 14 \mathrm{Ps}+14 \mathrm{H})$ \\
\hline 0.3 & -1.161 & -1.161 & -1.161 & -1.167 & -0.645 & -0.645 & -0.645 & -0.624 \\
0.4 & -1.446 & -1.446 & -1.446 & -1.453 & -0.849 & -0.849 & -0.850 & -0.838 \\
0.5 & -1.678 & -1.677 & -1.677 & -1.685 & -1.041 & -1.040 & -1.040 & -1.037 \\
0.6 & -1.858 & -1.857 & -1.857 & -1.867 & -1.216 & -1.214 & -1.215 & -1.213 \\
0.7 & -1.965 & -1.963 & -1.964 & -1.992 & -1.374 & -1.372 & -1.373 & -1.367 \\
\hline \hline
\end{tabular}

Hamiltonian in Eq. (2) should be changed to

$$
H=-\frac{1}{2} \sum_{i, j=1}^{3} \Lambda_{i j} \nabla_{i} \cdot \nabla_{j}+\sum_{i=1}^{3} \frac{Q q_{i}}{r_{i}}+\sum_{i, j=1}^{3} \frac{q_{i} q_{j}}{\left|\mathbf{r}_{j}-\mathbf{r}_{i}\right|}
$$

where $\Lambda_{i j}=\sum_{k=1}^{4} U_{i k} U_{j k} \frac{1}{m_{k}}, m_{k}=1$ in this work, and $U$ is a $4 \times 4$ transformation matrix [48]. The second modification is that the confining potential in Eq. (4) should include the degrees of freedom between the center-of-mass of $\mathrm{H}$ and the center-of-mass of Ps. The third modification is that the reduced mass $\mu$ in Eq. (6) should be changed to 1.997825085 , where the hydrogen nuclear mass is taken to be 1836.15267245 [49]. For $C_{6}$, however, we still use the infinite nuclear mass value, because its effect on the long-range polarization potential is negligibly small.

\section{RESULTS}

\section{A. Phase shifts}

Tables I and II list, respectively, the $S$ - and $P$-wave phase shifts obtained by the present CVM with $R_{0}=15$, and a comparison with the results from the complex Kohn (cKohn) method [25], the Kohn method [22], and the close-coupling (CC) method $[30,35]$, where $\delta_{L}^{+}$stands for the phase shift of the singlet spin configuration of the $L$-wave, and $\delta_{L}^{-}$for the triplet. Since the $S$-wave phase shifts at $k=0.1$ and 0.2 have been reported by Zhang et al. [41], here we only show the $S$-wave results at $k \geqslant 0.3$. Note that the cKohn phase shifts given in Tables I and II are extrapolated using the empirical formula $\tan \delta_{L}^{ \pm}(\omega)=\tan \delta_{L}^{ \pm}(\omega \rightarrow \infty)+c / \omega^{p}$, where $\omega$ is a non-negative integer that determines the maximum number of terms in the basis set, and $c$ and $p$ are parameters. For our CVM phase shifts in Tables I and II, the overall level of convergence of the calculated values (with $N=1800$, where $N$ is the size of basis set) is better than $0.16 \%$ for $S$-wave scattering and $0.40 \%$ for $P$-wave scattering. It can be seen that an excellent agreement has been achieved for all the $S$ and $P$-wave phase shifts among the CVM, cKohn method, and Kohn method. However, some discrepancies can be found between the CVM and CC results, with the largest being $4.0 \%$ for $\delta_{0}^{-}$at $k=0.3$ and $44.6 \%$ for $\delta_{1}^{-}$at $k=0.1$. In general, the $S$-wave phase shifts of the CC method have better agreement with the CVM values than the $P$-wave phase shifts, which may be due to the fact that more pseudostates are contained in the $S$-wave calculations.

Table III shows a comparison of the $D$-wave phase shifts among the present CVM, the cKohn method [25], and the $\mathrm{CC}$ method $[30,35]$. The short-range mixed-symmetry terms are not included in the trial wave functions of Woods et al. [25]. The cKohn results of $\delta_{2}^{+}$at $k=0.2-0.7$ are obtained by extrapolating to $\omega \rightarrow \infty$, while $\delta_{2}^{+}$at $k=0.1$ and all $\delta_{2}^{-}$ are obtained from $\omega=6$. The percentage difference in phase shift between $\omega \rightarrow \infty$ and $\omega=6$ is $6 \%$ for $\delta_{2}^{+}$at $k=0.2$, and it is less than $2 \%$ for $\delta_{2}^{+}$at $k=0.3-0.7$. For $\delta_{2}^{-}$the percentage difference between $\omega \rightarrow \infty$ and $\omega=6$ is $140 \%$ at $k=0.1$, and it is less than $25 \%$ at $k=0.2-0.7$, except for $k=0.4$, where it is $40 \%$. For our CVM $D$-wave phase shifts in Table III, the overall level of convergence is better than $0.3 \%$ at $k=0.1-0.2$, while it ranges from $0.6 \%$ to $1.3 \%$ at $k=0.3-0.7$. Table IV shows a convergence test of our $\delta_{2}^{-}$ at $k=0.4$, as the size of the basis set $N$ increases. One can see that for $N=1800$ the phase shift is converged to the second significant digit. The extrapolated cKohn phase shifts are close to the CVM values, with the percentage difference ranging from $1.8 \%$ to $2.5 \%$. There is, however, divergence between the cKohn phase shifts at $\omega=6$ and the corresponding CVM values, with the percentage difference ranging from

TABLE II. Comparison of $P$-wave phase shifts (in radians) among the present confined variational method (CVM), the complex Kohn method (cKohn) [25], and the close-coupling method (CC, [30] for singlet and [35] for triplet), where $\delta_{1}^{+}$stands for spin singlet, $\delta_{1}^{-}$stands for spin triplet, and $a^{b} \equiv a \times 10^{b}$.

\begin{tabular}{lllclll}
\hline \hline$k$ & $\delta_{1}^{+}(\mathrm{CVM})$ & $\delta_{1}^{+}(\mathrm{cKohn})$ & $\delta_{1}^{+}\left(\mathrm{CC}, 9 \mathrm{Ps}+9 \mathrm{H}+\mathrm{H}^{-}\right)$ & $\delta_{1}^{-}(\mathrm{CVM})$ & $\delta_{1}^{-}(\mathrm{cKohn})$ & $\delta_{1}^{-}(\mathrm{CC}, 9 \mathrm{Ps}+9 \mathrm{H})$ \\
\hline 0.1 & $0.228^{-1}$ & $0.227^{-1}$ & $0.221^{-1}$ & $-0.172^{-2}$ & $-0.172^{-2}$ & $-0.953^{-3}$ \\
0.2 & 0.192 & 0.192 & 0.183 & $-0.165^{-1}$ & $-0.165^{-1}$ & $-0.122^{-1}$ \\
0.3 & 0.612 & 0.611 & 0.580 & $-0.540^{-1}$ & $-0.540^{-1}$ & $-0.456^{-1}$ \\
0.4 & 0.997 & 0.996 & 0.956 & -0.114 & -0.114 & -0.104 \\
0.5 & 1.143 & 1.142 & 1.106 & -0.182 & -0.182 & -0.178 \\
0.6 & 1.164 & 1.163 & 1.134 & -0.247 & -0.246 & -0.247 \\
0.7 & 1.154 & 1.154 & 1.133 & -0.289 & -0.288 & -0.295 \\
\hline \hline
\end{tabular}


TABLE III. Comparison of $D$-wave phase shifts (in radians) among the present confined variational method (CVM), the complex Kohn method (cKohn) [25], and the close-coupling method (CC, [30] for singlet and [35] for triplet), where $\delta_{2}^{+}$stands for spin singlet, $\delta_{2}^{-}$stands for spin triplet, and $a^{b} \equiv a \times 10^{b}$.

\begin{tabular}{|c|c|c|c|c|c|c|}
\hline$k$ & $\delta_{2}^{+}(\mathrm{CVM})$ & $\delta_{2}^{+}(\mathrm{cKohn})$ & $\delta_{2}^{+}\left(\mathrm{CC} 9 \mathrm{Ps}+9 \mathrm{H}+\mathrm{H}^{-}\right)$ & $\delta_{2}^{-}(\mathrm{CVM})$ & $\delta_{2}^{-}(\mathrm{cKohn})$ & $\delta_{2}^{-}(\mathrm{CC} 9 \mathrm{Ps}+9 \mathrm{H})$ \\
\hline 0.1 & $1.64^{-4}$ & $1.36^{-4}$ & $2.02^{-4}$ & $9.46^{-5}$ & $5.81^{-5}$ & $8.48^{-5}$ \\
\hline 0.2 & $3.11^{-3}$ & $3.18^{-3}$ & $3.49^{-3}$ & $8.13^{-4}$ & $7.12^{-4}$ & $1.15^{-3}$ \\
\hline 0.3 & $1.65^{-2}$ & $1.62^{-2}$ & $1.73^{-2}$ & $1.51^{-3}$ & $1.10^{-3}$ & $2.84^{-3}$ \\
\hline 0.4 & $5.15^{-2}$ & $5.04^{-2}$ & $5.22^{-2}$ & $-4.52^{-4}$ & $-1.80^{-3}$ & $2.37^{-3}$ \\
\hline 0.5 & $1.17^{-1}$ & $1.14^{-1}$ & $1.16^{-1}$ & $-7.50^{-3}$ & $-1.07^{-2}$ & $-4.66^{-3}$ \\
\hline 0.6 & $2.14^{-1}$ & $2.09^{-1}$ & $2.08^{-1}$ & $-2.00^{-2}$ & $-2.54^{-2}$ & $-1.85^{-2}$ \\
\hline 0.7 & $3.40^{-1}$ & $3.33^{-1}$ & $3.24^{-1}$ & $-3.34^{-2}$ & $-4.28^{-2}$ & $-3.27^{-2}$ \\
\hline
\end{tabular}

$12 \%$ to $298 \%$. Another interesting point is that for the CVM and cKohn method, $\delta_{2}^{-}$changes sign from positive at $k=0.3$ to negative at $k=0.4$, whereas for the $\mathrm{CC}$ method it changes sign from positive at $k=0.4$ to negative at $k=0.5$, implying that the revealed $D$-wave interaction is more repulsive for the $\mathrm{CC}$ method than for the CVM and cKohn method.

The effect of the short-range mixed-symmetry terms has been investigated by Van Reeth and Humberston in their study of $e^{-}-\mathrm{H}$ scattering, and it has been found to be more important for the singlet than for the triplet $D$-wave phase shifts $[25,50]$. On the other hand, through a comparison between the CVM and cKohn results in Table III, one can see that the short-range mixed-symmetry terms are more important for the triplet than for the singlet. It is noted that these terms are included in our CVM calculations. To better understand this phenomenon, we calculate the singlet and triplet $D$-wave phase shifts at $k=0.1$ by excluding the terms. The obtained phase shifts at $k=0.1$ are $1.38 \times 10^{-4}$ for $\delta_{2}^{+}$and $7.72 \times 10^{-5}$ for $\delta_{2}^{-}$, which are larger than the corresponding cKohn values. Thus, taking the short-range mixed-symmetry terms into consideration increases the phase shifts by $18.8 \%$ and $22.5 \%$, respectively. In addition, we see that the absolute values of the $D$-wave phase shifts are smaller than those of the $S$ - and $P$-wave phase shifts, and they are more sensitive to the choice of the basis functions. The nonlinear parameters of the basis functions appear to be $k$-dependent and should be carefully optimized.

\section{B. Distortion effects}

In an atomic scattering process, the wave functions of the target and projectile will be distorted when they get close to each other. A quantitative study of the distortion effects is difficult but important [51]: For example, in probing material defects, the radius of the Ps confined in small pores is a crucial

TABLE IV. Convergence test of $\lambda$ in Eq. (7), total energy $E$ of Ps$\mathrm{H}$ scattering, and $D$-wave $\delta_{2}^{-}$(in radians) at $k=0.4$, i.e., $E=-0.71$, for the case of infinite nuclear mass, as the size of the basis set $N$ increases (in atomic units; $a^{b} \equiv a \times 10^{b}$ ).

\begin{tabular}{lccc}
\hline \hline$N$ & $\lambda$ & $E$ & $\delta_{2}^{-}$ \\
\hline 1200 & $1.24260525^{-4}$ & -0.70999994 & $-0.482^{-3}$ \\
1400 & $1.24261425^{-4}$ & -0.70999993 & $-0.463^{-3}$ \\
1600 & $1.24261755^{-4}$ & -0.70999992 & $-0.455^{-3}$ \\
1800 & $1.24261925^{-4}$ & -0.70999993 & $-0.452^{-3}$ \\
\hline \hline
\end{tabular}

parameter for understanding the complicated annihilationlifetime spectroscopy $[13,52]$. In all the existing calculations of Ps-H scattering, the distortion effects are handled by two methods: One is the expansion of the scattering wave function in terms of correlated short-range basis functions, and one is the inclusion of pseudostates describing Ps, $\mathrm{H}$, and $\mathrm{H}^{-}$. In Table III, for example, nine pseudostates of Ps, nine pseudostates of $\mathrm{H}$, and one pseudostate of $\mathrm{H}^{-}$are included in the CC calculations. In this work, we define three distortion distances $R_{d}^{\mathrm{H}}, R_{d}^{\mathrm{Ps}}$, and $R_{d}^{\mathrm{Ps}-\mathrm{H}}$, where $R_{d}^{\mathrm{H}}$ and $R_{d}^{\mathrm{Ps}}$ are measures of the distance between Ps and $\mathrm{H}$ when the distortion of $\mathrm{H}$ and Ps can be ignored, respectively, and $R_{d}^{\mathrm{Ps}-\mathrm{H}}$ is a measure of this distance when the distortion of both $\mathrm{H}$ and Ps can be ignored so that the long-range polarization potential dominates. Since the polarizability of Ps is larger than that of $\mathrm{H}$, the distortion of Ps dominates over that of $\mathrm{H}$, indicating $R_{d}^{\mathrm{Ps}}>R_{d}^{\mathrm{H}}$. Thus, we have $R_{d}^{\mathrm{Ps}-\mathrm{H}}=\max \left(R_{d}^{\mathrm{H}}, R_{d}^{\mathrm{Ps}}\right)=R_{d}^{\mathrm{Ps}}$.

The distortion distance $R_{d}^{\mathrm{Ps}}$ for Ps can be calculated as follows. For the electron, the probability density distribution is

$$
f_{e}^{i}(R)=\int d \Omega_{\boldsymbol{R}}\left\langle\Psi\left|\delta\left(\mathbf{r}_{i}-\boldsymbol{R}\right)\right| \Psi\right\rangle R^{2},
$$

where $i=1,2,\langle\cdots\rangle$ means the integration over the relative coordinates $\mathbf{r}_{1}, \mathbf{r}_{2}$, and $\mathbf{r}_{3}$, and $\int d \Omega_{\boldsymbol{R}} \cdots$ is the integration over the solid angle of $\boldsymbol{R} . f_{e}^{i}(R)$ can be evaluated, since the matrix element for the Dirac $\delta$ function can be analytically calculated using the ECG basis. For the two electrons, we have $f_{e}^{1}(R)=f_{e}^{2}(R) \equiv f_{e}(R)$. For the positron, the probability density distribution is

$$
f_{e^{+}}(R)=\int d \Omega_{R}\left\langle\Psi\left|\delta\left(\mathbf{r}_{3}-\boldsymbol{R}\right)\right| \Psi\right\rangle R^{2} .
$$

The distortion distance $R_{d}^{\mathrm{Ps}}$ can be obtained according to

$$
f_{e^{+}}(R)=2 f_{e}(R), \quad R \geqslant R_{d}^{\mathrm{PS}}
$$

because of the electric neutrality requirement of Ps at $R \geqslant R_{d}^{\mathrm{Ps}}$.

A comparison of the probability density distributions for singlet scattering at $k=0.1$ is shown in Fig. 1. We find for $f_{e}(R)$ with $R<5$ high peaks for $S$-, $P$-, and $D$-wave scattering. For $f_{e^{+}}(R)$ with $R<5$, however, due to the repulsive interaction between the positron and the hydrogen nucleus, there is a low peak for $S$-wave scattering and no peak for $P$ - and $D$-wave scattering. For $R>5$, the probability density distributions of the electron and positron become similar. In this work, $R_{d}^{\mathrm{Ps}}$ is 


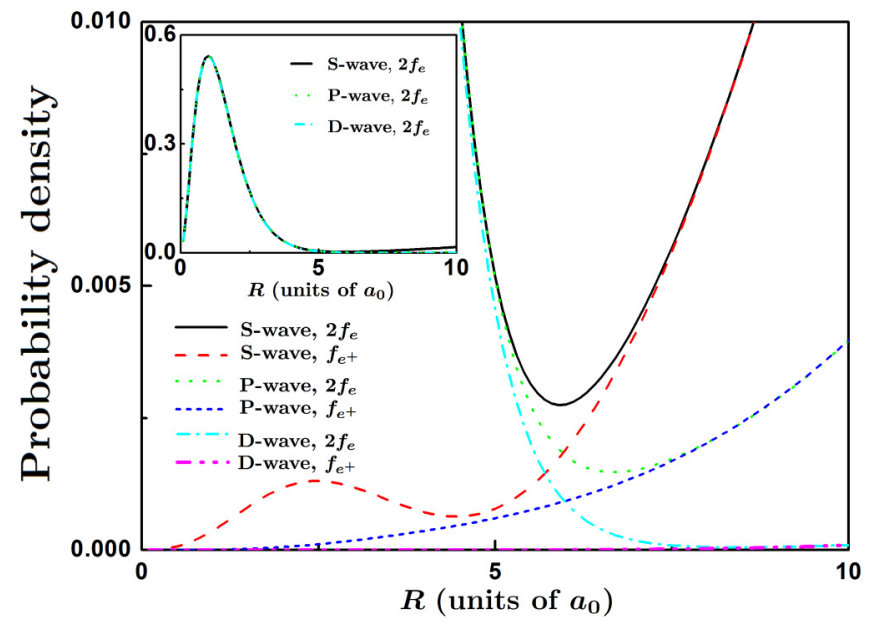

FIG. 1. Probability density distributions $f_{e}(R)$ and $f_{e^{+}}(R)$ for $S$-, $P$-, and $D$-wave singlet Ps-H scattering at $k=0.1 a_{0}^{-1}$, where $a_{0}$ is the Bohr radius.

determined according to the criterion

$$
\left|\frac{f_{e^{+}}(R)-2 f_{e}(R)}{f_{e^{+}}(R)}\right|_{R=R_{d}^{\mathrm{Ps}}}<1 \% .
$$

This yields $R_{d}^{\mathrm{Ps}}$ to be 7.7, 7.8, and 10.0 for $S$-, $P$-, and $D$-wave singlet scattering at $k=0.1$, respectively, which indicates that $R_{d}^{\mathrm{Ps}}$ increases with the total orbital angular momentum $L$ for the same $k$ and spin configuration. $R_{d}^{\mathrm{Ps}}$ is determined analogously for other $k, L$, and spin configurations. We find for the same $L$ and spin configuration that $R_{d}^{\text {Ps }}$ decreases as the Ps momentum $k$ increases. For example, for $L=0$ and singlet scattering we obtain $R_{d}^{\mathrm{Ps}}=7.7,7.3,6.8,6.7,6.5,6.2$, and 5.7 at $k=0.1-0.7$ with increments of $0.1 . R_{d}^{\mathrm{Ps}}$ of singlet and triplet scattering is similar for the same $k$ and $L$.

\section{C. $S$-wave scattering lengths}

Though the CVM scattering phase shifts $\delta_{0}^{ \pm}$at $k=0.1$ and 0.2 have been reported by Zhang et al. [41], the CVM scattering lengths have not been reported. In this work, the $S$-wave scattering lengths are obtained by fitting the calculated phase shifts to the effective range theory,

$$
k \cot \delta_{0}^{ \pm}=-\frac{1}{a^{ \pm}}+\frac{r_{0}^{ \pm} k^{2}}{2}
$$

where $a^{+}$and $a^{-}$are, respectively, the singlet and triplet $S$-wave scattering lengths, and $r_{0}^{ \pm}$are the corresponding effective ranges. If we take into account the long-range van der Waals interaction, we should use the modified formula of Flannery et al. [53],

$$
k \cot \delta_{0}^{ \pm}=-\frac{1}{a^{ \pm}}+\frac{r_{0}^{ \pm} k^{2}}{2}-\frac{4 \pi C_{6} k^{3}}{15\left(a^{ \pm}\right)^{2}}-\frac{16 C_{6}}{15 a^{ \pm}} k^{4} \ln k .
$$

These two effective range formulas are more suitable for low$k$ scattering so that higher-order terms in the expansions can be neglected.

To extract more accurate scattering lengths in the case of infinite nuclear mass (INM), the $S$-wave phase shifts at $k=$ 0.04-0.08 are calculated, and the results are given in Table V.
TABLE V. Singlet $\delta_{0}^{+}$and triplet $\delta_{0}^{-}$(in radians) $S$-wave phase shifts at $k=0.04-0.08$ for the cases of infinite nuclear mass (INM) and finite nuclear mass (FNM).

\begin{tabular}{ccccc}
\hline \hline$k$ & $\delta_{0}^{+}(\mathrm{INM})$ & $\delta_{0}^{+}(\mathrm{FNM})$ & $\delta_{0}^{-}(\mathrm{INM})$ & $\delta_{0}^{-}(\mathrm{FNM})$ \\
\hline 0.04 & $-1.7107^{-1}$ & $-1.7124^{-1}$ & $-8.4648^{-2}$ & $-8.4653^{-2}$ \\
0.05 & $-2.1532^{-1}$ & $-2.1553^{-1}$ & $-1.0686^{-1}$ & $-1.0687^{-1}$ \\
0.06 & $-2.5801^{-1}$ & $-2.5826^{-1}$ & $-1.2833^{-1}$ & $-1.2834^{-1}$ \\
0.07 & $-3.0049^{-1}$ & $-3.0078^{-1}$ & $-1.4983^{-1}$ & $-1.4985^{-1}$ \\
0.08 & $-3.4272^{-1}$ & $-3.4308^{-1}$ & $-1.7138^{-1}$ & $-1.7140^{-1}$ \\
\hline \hline
\end{tabular}

Table VI shows a convergence test for $\delta_{0}^{+}$at $k=0.05$, as the size of the basis set $N$ increases. One can see that for $N=1800$, the phase shift is converged to the fifth significant digit. The scattering lengths determined by Eqs. (14) and (15) are given in Table VII, together with a comparison with other theoretical values. We can see that the CVM scattering lengths obtained by Eqs. (14) and (15) are very close to each other, whereas the CVM effective ranges are not, indicating that the addition of the $k^{3}$ and $k^{4} \ln k$ terms from the van der Waals interaction has little effect on $a^{ \pm}$but a significant effect on $r_{0}^{ \pm}$. There are also noticeable differences between our CVM values and the cKohn values of $1.1 \%$. In addition, the cKohn results are closer to those of the SVM. In general, it is preferable to extract the scattering lengths from the low$k$ range, where the complicated short-range correlations are more important. Thus, the nonlinear parameters in the basis functions must be carefully optimized for each value of $k$, as we did for $k=0.04-0.08$.

All previous Ps-H scattering calculations have been performed under the INM approximation. The finite nuclear mass (FNM) effect is studied here in detail, and the phase shifts and scattering lengths obtained are shown in Tables V and VII, respectively. An important advantage of the present calculation method is that the Hamiltonian and its matrix elements in the FNM case preserve their functional form under the general linear transformation $U$ entering Eq. (9). Thus, the extension from INM to FNM is natural but results in additional computational complexity. Comparing the FNM and INM phase shifts in Table $\mathrm{V}$, we find that the FNM effect is more important for singlet than for triplet scattering with percentage differences of $0.1 \%$ and $0.01 \%$, respectively. This can be explained by the $S$-wave probability density distributions $f_{\mathrm{ee}^{+}}(R)$ and $f_{\mathrm{e}^{+}}(R)$, ee $\mathrm{e}^{+}$denoting the center-of-mass of the electron-positron pair, for singlet and triplet scattering at $k=0.05$ shown in Fig. 2. We find low peaks at $R<5$ for

TABLE VI. Convergence test of $\lambda$ in Eq. (7), total energy $E$ of Ps-H scattering, and $S$-wave $\delta_{0}^{+}$(in radians) at $k=0.05$, i.e., $E=$ -0.749375 , for the case of infinite nuclear mass, as the size of the basis set $N$ increases (in atomic units, $a^{b} \equiv a \times 10^{b}$ ).

\begin{tabular}{lccc}
\hline \hline$N$ & $\lambda$ & $E$ & $\delta_{0}^{+}$ \\
\hline 1200 & $2.95617617^{-7}$ & -0.749374994 & -0.21539 \\
1400 & $2.95631417^{-7}$ & -0.749374994 & -0.21535 \\
1600 & $2.95640417^{-7}$ & -0.749374995 & -0.21532 \\
1800 & $2.95642417^{-7}$ & -0.749374995 & -0.21532 \\
\hline \hline
\end{tabular}


TABLE VII. $S$-wave scattering lengths ( $a^{+}$for singlet and $a^{-}$for triplet) and effective ranges ( $r_{0}^{+}$for singlet and $r_{0}^{-}$for triplet) for the cases of infinite nuclear mass (INM) and finite nuclear mass (FNM), obtained by the present confined variational method (CVM), the complex Kohn method (cKohn) [25], and the stochastic variational method (SVM) [29], in atomic units.

\begin{tabular}{lccccl}
\hline \hline Model & $k$ & $a^{+}$ & $r_{0}^{+}$ & $a^{-}$ & \multicolumn{1}{c}{$r_{0}^{-}$} \\
\hline INM CVM Eq. (14) & $0.04-0.08$ & 4.2841 & 3.1032 & 2.1133 & 3.9563 \\
INM CVM Eq. (15) & $0.04-0.08$ & 4.2854 & 3.0645 & 2.1126 & 4.4759 \\
INM cKohn Eq. (14) & $0.1-0.5$ & 4.308 & 2.275 & 2.162 & 1.343 \\
INM cKohn Eq. (14) & $0.001-0.009$ & 4.331 & 2.197 & 2.137 & 2.035 \\
INM cKohn Eq. (15) & $0.001-0.009$ & 4.331 & 2.221 & 2.137 & 2.139 \\
INM SVM Eq. (14) & $0-0.5$ & 4.34 & 2.39 & 2.22 & 1.29 \\
FNM CVM Eq. (14) & $0.04-0.08$ & 4.2881 & 3.1117 & 2.1134 & 3.9694 \\
\hline \hline
\end{tabular}

singlet scattering, but not for triplet scattering. In the case of singlet scattering, the positron or electron-positron pair can get closer to the hydrogen nucleus and thus it is more sensitive to the nuclear mass effect than in the case of triplet scattering, which can be seen from Table VII, as the FNM effect increases the singlet scattering length by $0.1 \%$ but the triplet scattering length only by $0.005 \%$.

\section{SUMMARY}

Using the confined variational method combined with an explicitly correlated Gaussian basis, the phase shifts of ${ }^{1,3} S$-, ${ }^{1,3} \mathrm{P}$-, and ${ }^{1,3} \mathrm{D}$-wave Ps-H scattering below the Ps excitation threshold have been calculated. Our $S$ - and $P$-wave phase shifts agree well with the results of the complex Kohn method of Woods et al. The large discrepancy in the literature for $D$-wave scattering has been resolved. Comparing the probability density distributions of the positron and the electron, we have found that the distortion distance of Ps decreases with the Ps momentum and increases with the total orbital angular momentum. Using the effective range theory, the $S$ wave scattering lengths have been extracted at $k=0.04-0.08$

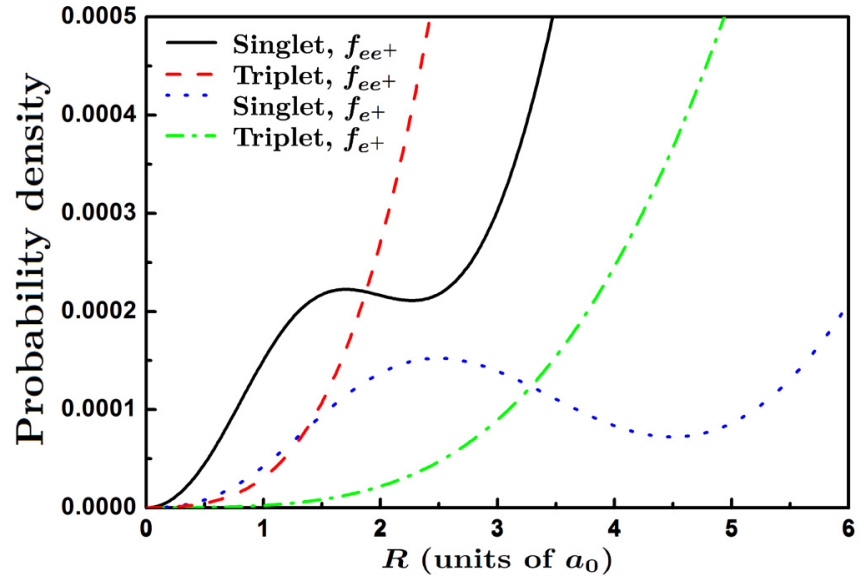

FIG. 2. Probability density distributions $f_{\mathrm{ee}^{+}}(R)$ and $f_{\mathrm{e}^{+}}(R), \mathrm{ee}^{+}$ denoting the center-of-mass of the electron-positron pair, for $S$-wave singlet and triplet Ps-H scattering at $k=0.05 a_{0}^{-1}$, where $a_{0}$ is the Bohr radius.

for both infinite and finite nuclear mass. In the case of infinite nuclear mass, the obtained scattering length is smaller than that predicted by the complex Kohn and stochastic variational methods. We have also demonstrated that the finite nuclear mass effect increases both the singlet and triplet scattering lengths, with a more significant impact in the case of singlet scattering.

\section{ACKNOWLEDGMENTS}

This work was supported by the National Natural Science Foundation of China under Grants No. 11704399 and No. 11934014, by the Strategic Priority Research Program of the Chinese Academy of Sciences under Grant No. XDB21030300, and by the National Key Research and Development Program of China under Grant No. 2017YFA0304402. The research reported in this publication was supported by funding from King Abdullah University of Science and Technology (KAUST). Z.-C. Y. was supported by NSERC of Canada.
[1] S. G. Karshenboim, Phys. Rep. 422, 1 (2005).

[2] T. Yamazaki, A. Miyazaki, T. Suehara, T. Namba, S. Asai, T. Kobayashi, H. Saito, I. Ogawa, T. Idehara, and S. Sabchevski, Phys. Rev. Lett. 108, 253401 (2012).

[3] G. S. Adkins, M. Kim, C. Parsons, and R. N. Fell, Phys. Rev. Lett. 115, 233401 (2015).

[4] C. Frugiuele, J. Pérez-Ríos, and C. Peset, Phys. Rev. D 100, 015010 (2019).

[5] L. Gurung, T. J. Babij, S. D. Hogan, and D. B. Cassidy, Phys. Rev. Lett. 125, 073002 (2020).

[6] D. B. Cassidy, V. E. Meligne, and A. P. Mills, Phys. Rev. Lett. 104, 173401 (2010).

[7] H. K. Avetissian, A. K. Avetissian, and G. F. Mkrtchian, Phys. Rev. Lett. 113, 023904 (2014).

[8] D. B. Cassidy, Eur. Phys. J. D 72, 53 (2018).

[9] A. P. Mills, Phys. Rev. A 100, 063615 (2019).
[10] C. M. Surko, M. Leventhal, and A. Passner, Phys. Rev. Lett. 62, 901 (1989).

[11] G. F. Gribakin, J. A. Young, and C. M. Surko, Rev. Mod. Phys. 82, 2557 (2010).

[12] M. J. Puska and R. M. Nieminen, Rev. Mod. Phys. 66, 841 (1994).

[13] F. Tuomisto and I. Makkonen, Rev. Mod. Phys. 85, 1583 (2013).

[14] C. Hugenschmidt, Surf. Sci. Rep. 71, 547 (2016).

[15] G. Gabrielse, R. Kalra, W. S. Kolthammer, R. McConnell, P. Richerme, D. Grzonka, W. Oelert, T. Sefzick, M. Zielinski, D. W. Fitzakerley, M. C. George, E. A. Hessels, C. H. Storry, M. Weel, A. Müllers, and J. Walz (ATRAP Collaboration), Phys. Rev. Lett. 108, 113002 (2012).

[16] A. S. Kadyrov, C. M. Rawlins, A. T. Stelbovics, I. Bray, and M. Charlton, Phys. Rev. Lett. 114, 183201 (2015). 
[17] A. S. Kadyrov, I. Bray, M. Charlton, and I. I. Fabrikant, Nat. Commun. 8, 1544 (2017).

[18] A. W. Alrowaily, S. J. Ward, and P. V. Reeth, J. Phys. B 52, 205201 (2019).

[19] C. M. DeMars, J. B. Kent, and S. J. Ward, Eur. Phys. J. D 74, 48 (2020).

[20] C. M. DeMars, S. J. Ward, J. Colgan, S. Amami, and D. H. Madison, Atoms 8, 26 (2020).

[21] S. J. Brawley, S. Armitage, J. Beale, D. E. Leslie, A. I. Williams, and G. Laricchia, Science 330, 789 (2010).

[22] P. V. Reeth and J. W. Humberston, J. Phys. B 36, 1923 (2003).

[23] P. Van Reeth and J. Humberston, Nucl. Instrum. Methods Phys. Res., Sect. B 221, 140 (2004).

[24] B. A. P. Page, J. Phys. B 9, 1111 (1976).

[25] D. Woods, S. J. Ward, and P. Van Reeth, Phys. Rev. A 92, 022713 (2015).

[26] D. Woods, arXiv:1508.05681.

[27] S. Chiesa, M. Mella, and G. Morosi, Phys. Rev. A 66, 042502 (2002).

[28] I. A. Ivanov, J. Mitroy, and K. Varga, Phys. Rev. Lett. 87, 063201 (2001).

[29] I. A. Ivanov, J. Mitroy, and K. Varga, Phys. Rev. A 65, 032703 (2002).

[30] H. Walters, A. Yu, S. Sahoo, and S. Gilmore, Nucl. Instrum. Methods Phys. Res., Sect. B 221, 149 (2004).

[31] P. K. Sinha, P. Chaudhury, and A. S. Ghosh, J. Phys. B 30, 4643 (1997).

[32] C. P. Campbell, M. T. McAlinden, F. G. R. S. MacDonald, and H. R. J. Walters, Phys. Rev. Lett. 80, 5097 (1998).

[33] S. K. Adhikari and P. K. Biswas, Phys. Rev. A 59, 2058 (1999).

[34] P. K. Sinha, A. Basu, and A. S. Ghosh, J. Phys. B 33, 2579 (2000).

[35] J. E. Blackwood, M. T. McAlinden, and H. R. J. Walters, Phys. Rev. A 65, 032517 (2002).
[36] J. E. Blackwood, M. T. McAlinden, and H. R. J. Walters, Phys. Rev. A 65, 030502(R) (2002).

[37] S. Hara and P. A. Fraser, J. Phys. B 8, L472 (1975).

[38] H. Ray and A. S. Ghosh, J. Phys. B 29, 5505 (1996).

[39] H. Ray and A. S. Ghosh, J. Phys. B 30, 3745 (1997).

[40] A. R. Swann, J. Phys.: Conf. Ser. 1412, 052009 (2020).

[41] J.-Y. Zhang, Z.-C. Yan, and U. Schwingenschlögl, Europhys. Lett. 99, 43001 (2012).

[42] J. Mitroy, J. Y. Zhang, and K. Varga, Phys. Rev. Lett. 101, 123201 (2008).

[43] J. Y. Zhang, J. Mitroy, and K. Varga, Phys. Rev. A 78, 042705 (2008).

[44] J.-Y. Zhang, M.-S. Wu, Y. Qian, X. Gao, Y.-J. Yang, K. Varga, Z.-C. Yan, and U. Schwingenschlögl, Phys. Rev. A 100, 032701 (2019).

[45] M.-S. Wu, J.-Y. Zhang, X. Gao, Y. Qian, H.-H. Xie, K. Varga, Z.-C. Yan, and U. Schwingenschlögl, Phys. Rev. A 101, 042705 (2020).

[46] D. W. Martin and P. A. Fraser, J. Phys. B 13, 3383 (1980).

[47] D. G. Meredith and P. A. Fraser, J. Phys. B 51, 055201 (2018).

[48] J. Mitroy, S. Bubin, W. Horiuchi, Y. Suzuki, L. Adamowicz, W. Cencek, K. Szalewicz, J. Komasa, D. Blume, and K. Varga, Rev. Mod. Phys. 85, 693 (2013).

[49] P. J. Mohr, B. N. Taylor, and D. B. Newell, Rev. Mod. Phys. 84, 1527 (2012).

[50] P. V. Reeth, J. W. Humberston, D. Woods, and S. J. Ward (unpublished).

[51] R. S. Wilde and I. I. Fabrikant, Phys. Rev. A 97, 052708 (2018).

[52] R. Brown, Q. Prigent, A. R. Swann, and G. F. Gribakin, Phys. Rev. A 95, 032705 (2017).

[53] M. R. Flannery, in Springer Handbook of Atomic, Molecular, and Optical Physics, 2nd ed., edited by G. W. F. Drake (Springer, New York, 2006), p. 668. 